\title{
Wenn die Experten gehen ...
}

\section{Was Organisationen und Unternehmen beim Weggang älterer Mitarbeiterinnen und Mitarbeiter bedenken sollten}

\author{
CHRISTIAN TAUDT \\ HEIDEMARIE GREGOR UND \\ IRMGARD SCHROLL-DECKER \\ Christian Taudt, Diplom-Ingenieur, \\ seit über 20 Jahren als Führungs- \\ kraft in der Entwicklung in der Auto- \\ mobilindustrie tätig; seit sechs Jah- \\ ren mit den Themen Wissens- und \\ Kompetenzmanagement betraut; \\ M.A.-Abschluss 2012, seither Work- \\ shopmoderation, Teamentwicklung \\ und Entwicklungsprozesse. \\ c-taudt@t-online.de
}

Heidemarie.Gregor, Akademische Rätin, Diplom-Pädagogin (Univ.), Diplom-Sozialpädogin (FH) ist seit 1995 wissenschaftliche Mitarbeiterin und Beauftragte für das Praktische Studiensemester an der Fakultät Angewandte Sozialwissenschaften der Hochschule Regensburg, Moderation von Workshops Leitbildprozessen und Weiterbildungsseminaren.

Heidemarie.Gregor@hs-regensburg.de

Prof. Dr. Irmgard Schroll-Decker, Professur für Sozialmanagement und Bildungsarbeit, lehrt an der Fakultät Angewandte Sozialwissenschaften der Hochschule Regensburg. Irmgard.Schroll-Decker@ hs-regensburg.de

\author{
Wenn ältere Mitarbeiterinnen und Mitarbeiter \\ in den Ruhestand wechseln, können wertvolle \\ Wissensbestände und Kontakt der Organisation \\ oder dem Unternehmen verloren gehen. Damit \\ mehr zurückbleibt als ein leerer Stuhl, bietet sich die \\ Durchführung eines Leaving-Expert-Prozesses an.
}

Ein Blick auf die bundesdeutsche Alterspyramide lässt erkennen, welche Brisanz im demografischen Wandel liegt (vgl. Statistisches Bundesamt, 2009). Die deutsche Volkswirtschaft wird im Wesentlichen von Personen getragen, die in der zweiten Hälfte ihres Erwerbslebens stehen (vgl. Fischer, 2007). Viele der erfahrenen und leistungstragenden Mitarbeiterinnen und Mitarbeiter werden in den nächsten Jahren in Rente gehen.

Auf die Unternehmen kommen mehrere Herausforderungen zu. Zum einen wird es zunehmend schwierig, die Lücken mit genügend und geeigneten Nachwuchskräften aufzufüllen. Zum anderen müssen sie sich auf eine zunehmend ältere Belegschaft, deren spezifische Bedürfnisse und eine Welle von altersbedingt ausscheidenden Beschäftigten einstellen. Beide Problemfelder sind mittlerweile ins Bewusstsein der betrieblichen Personalabteilungen gerückt (vgl. Seyfried 2011 und Morschhäuser 2006). Angemessene Gestaltungsoptionen werden empfohlen, beispielsweise hinsichtlich betrieblicher Weiterbildung, Ergonomie, gesundheitsbewusster Personalarbeit, Vereinbarkeit von Pflege und Berufstätigkeit etc.

Darüber hinaus wird die Notwendigkeit einer "Wissensübergabe « der älteren Mitarbeitenden an die Nachfolger thematisiert. Die meisten Autoren und Referentinnen brechen jedoch entweder an der Stelle ab, an der die Ausgestaltung eines solchen Transfers konkret werden müsste, oder sie legen den Schwerpunkt auf die Explizierbarkeit von Wissen - und »übersehen« damit das Problem, dass sich Erfahrung und Kompetenzen nicht in Datenbanken transferieren lassen.

\section{Wissenstransfer bei Verrentung sicherstellen}

Im folgenden Beitrag wird ein Modell entwickelt, welches den Wissenstransfer zwischen dem abgehenden Mitarbeiter (im Folgenden als »Leaving-Expert « bezeichnet) und den Nachfolgern optimiert. Statt jedoch nur auf die Reduzierung des Wissensverlustes zu fokussieren, kann der »Leaving-Expert-Prozess « wertschöpfend gestaltet werden, also Zusatznutzen für die Organisation kreieren. Um beides zu erreichen, sind folgende Fragen zu betrachten:

- Wie kann das zu transferierende Wissen systematisch erfasst werden?

- Wie sind Wissenstransfer und Lernprozesse bei den Nachfolgerinnen und Nachfolgern effizient zu gestalten? Wie können Kompetenzaufbau und Erfahrungslernen optimiert werden?

- Welche Faktoren beeinflussen die Motivation der Beteiligten? Wissenstransfer ist vor allem ein sozialer Prozess - welche Gestaltungsmöglichkeiten für einen erfolgreichen Wissenstransfer ergeben sich aus dieser Sichtweise?

- Wie kann das Team der Kolleginnen und Kollegen auf die Veränderungen vorbereitet werden, die mit dem Weggang eines Erfahrungsträgers verbunden sind? Wie kann dieser Schritt mittels Teamentwicklungs- 


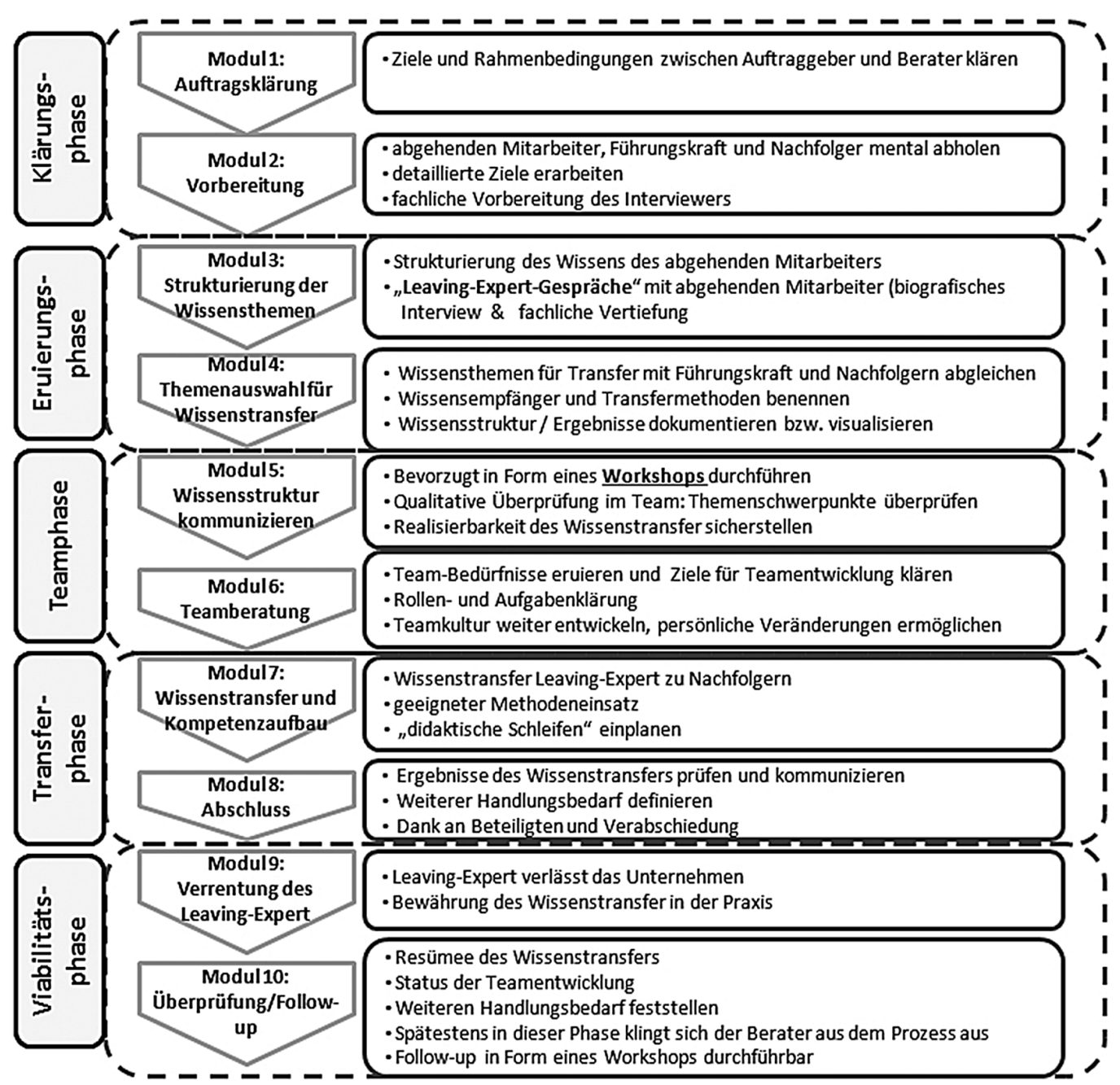

Abb. 1: Wenn ältere Mitarbeiterinnen und Mitarbeiter in Rente gehen, sollte der Wissenstransfer strukturiert vonstatten gehen.

maßnahmen begleitet werden und der Teamleistung förderlich sein?

- Wie können die einzelnen Schritte zu einem Gesamtprozess strukturiert werden?

Der im nachfolgenden dargestellte Ablauf ist als »idealer « Prozess zu verstehen, der in der betrieblichen Praxis an die entsprechenden Gegebenheiten adaptiert werden muss. Ausgelöst wird der Prozess, wenn ein erfahrener betrieblicher Experte das Unternehmen aus Altersgründen verlassen wird, und der Betrieb in dieser Situation ein zielgerichtetes Interesse am Erhalt und der Weitergabe des Wissens und der Kompetenzen dieser Mitarbeiterin oder dieses Mitarbeiters hat.

Eine spezifischere Beschreibung von Expertenstatus und Erfahrungswissen (wie exemplarisch bei Schulz-Wild \& Böhle 2006, und Neuweg 2004 aufgezeigt) ist zur Initiierung des Prozesses nicht notwendig. Um den Prozess möglichst professionell durchzuführen, ist anzuraten, einen internen oder externen Prozessberater einzubinden, der die entsprechenden Methoden kompetent anwenden kann. $\mathrm{Zu}$ dessen Aufgaben gehört beispielsweise das Führen von Expertengesprächen, um die Explikation der Wissensgebiete und die Reflexion von latentem Erfahrungswissen zu ermöglichen. Neben dem methodischen Know-how und ausgeprägten sozialen Kompetenzen benötigt der Prozessberater auch inhaltliches Fachwissen, um »die Sprache des Experten« zu verstehen und die Akzeptanz des Experten und Effizienz der fachlich orientierten Gespräche zu ermöglichen.

Damit der Wissenstransfer von abgehenden Expertinnen und Experten nicht der Beliebigkeit anheimgestellt ist, richten immer mehr Unternehmen ihr Augenmerk auf die Erstellung eines Gesamtkonzeptes und eine Standardi- sierung des Vorgehens. Ein detailliertes "normatives Leaving-Expert-Konzept « hat Taudt (vgl. 2013) entwickelt. Nachfolgend wird es in seiner Grundstruktur vorgestellt. Anpassungen an die jeweiligen betrieblichen Gegebenheiten und die fallspezifische Situation sind einzelbetrieblich zu leisten. Der Prozess ist in fünf Phasen strukturiert, die nachfolgend beschrieben werden.

Die einzelnen Phasen sind in jeweils zwei Module unterteilt, in denen die als zentral herauskristallisierten Aufgaben bearbeitet werden (vgl. Abb. 1). Als Rahmenbedingung wird für das Konzept angenommen, dass der Prozess von einem oder mehreren internen oderexternen Beratern begleitet wird.

\section{Die fünf Phasen des Leaving- Expert-Konzepts}

In der Klärungsphase werden nicht nur die eigentliche Zielsetzung, sondern auch 
die organisatorischen Rahmenbedingungen, der Zeitrahmen, das Budget, die Beteiligten und deren Kommunikation untereinander geklärt. Verantwortlich für die Klärung ist die Führungskraft des abgehenden Mitarbeiters. Weitere Beteiligte sind üblicherweise der Prozessberater und Personalabteilung. Auch der abgehende Mitarbeiter und sein Nachfolger können eingebunden werden.

In der Eruierungsphase klärt sich, welche Wissensgebiete und fachliche Vertiefung als für den Prozess relevant ausgewählt werden. Mit dem abgehenden Mitarbeiter können - sofern der Betroffene zustimmt - biografischorientierte Gespräche geführt werden, die sich auf den beruflichen Werdegang und die für den Übergabeprozess für Betrieb und Mitarbeiter sensiblen Themen beziehen. Die obligatorische fachlich-inhaltliche Vertiefung fokussiert die Kernfragen: »Welches Wissen soll transferiert werden? « »Wer sind die Wissensempfänger? « "Welche Transfermethoden sind geeignet? « Die Antworten hierauf können in einem Wissensstrukturplan zusammengefasst werden.

Zentrales Anliegen in der Klärungsund Eruierungsphase ist die Vertrauensbildung, so dass sich die abgehende Mitarbeiterin oder der abgehende Mitarbeiter auf den Prozess einlassen kann, ihn für sich und den Betrieb als sinnvoll und hilfreich erlebt. Charakteristisch für das Leaving-Expert-Gespräch sind ein echtes Interesse, Empathie und ein sensibler Umgang mit möglichen Blockaden. Hier wird auch das weitere Verfahren geregelt, das heißt: Wie vertraulich wird mit den Daten umgegangen, welche latenten Bedürfnisse und mögliche Bedenken sind vorhanden?

Die Einbindung der betroffenen Kollegen in der anschließenden Teamphase soll zum einen Transparenz über die bisherigen Ergebnisse geben, um offene Punkte und Fragen klären zu können, zum anderen ist das Team "auf die Zeit danach" vorzubereiten. Rollenund Aufgabenverteilungen, Anpassung von Entscheidungs- und Kommunikationsprozessen können dabei betrachtet werden. Es bietet sich an, diese Phase mittels eines spezifischen Workshops zu gestalten. Dieser kann als Rahmen genutzt werden für wichtige symbolische Handlungen: Danksagung und Wertschätzung der beruflichen Leistung, Übergabe an die Nachfolger.
Die anschließende Transferphase beinhaltet den eigentlichen Wissenstransfer und Kompetenzaufbau. Hier ist die richtige Auswahl der jeweils geeigneten Methode maßgeblich dafür, ob der Transfer gelingt und die Nachfolgerin oder der Nachfolger entsprechende Kompetenzen aufbaut. Die vorherige Festlegung der Lernziele ermöglicht nicht nur einen zielgerichteten Transfer, sondern ebenso eine Erfolgskontrolle und weiteren Handlungsbedarf rechtzeitig indizieren zu können.

In der Viabilitätsphase verlässt der Leaving-Expert das Unternehmen und die Übergabe muss sich in der Praxis bewähren. Nach etwa einem halben Jahr kann ein Resümee gezogen werden. Weiterer Handlungsbedarf kann beispielsweise in einem Follow-up-Workshop im Team besprochen werden. Der Berater beendet, wenn nicht bereits vorher geschehen, seine Begleitung.

Das inhaltliche und transferdidaktische Vorgehen in den einzelnen Phasen ist unterschiedlich.

- Klärungsphase: Zur Klärungsphase gehören die Module Auftragsklärung und Vorbereitung. Auftraggeber kann die Geschäftsleitung, die Personalabteilung oder die direkte Führungskraft des Leaving-Experts sein. Die Perspektiven und Wünsche der Auftraggeber sind mit den Möglichkeiten des Beraters abzugleichen. Je nach einzelnem Leaving-Expert-Fall klärt sich hier, wer am Prozess teilnimmt (Führungskraft, abgehende Person, Teammitglieder oder Schnittstellenbetroffene) und entsprechend informiert wird. Ist ein parallel verlaufender Team- und Organisationsentwicklungsprozess intendiert, sind in dieser Phase auch Interviews mit Teammitgliedern und Schnittstellenpartnern zu platzieren. Als Resultat dieser Interventionen sind eine genaue Zielformulierung und die Rückmeldung an die Führungskraft festzuhalten. In der Klärung sind zwei Ebenen zu unterscheiden: Zum einen können Ziele und Vorgehen für einzelne Fälle besprochen werden. Zum anderen kann das Konzept für einen betriebsweiten Einsatz des LeavingExpert Prozesses erörtert werden.

- Eruierungsphase: In dieser Phase werden die Wissensstruktur des Leaving-Expert eruiert und die für den Transfer notwendigen Themen ausgewählt. Für beide Schritte sind Einzelgespräche die geeignete $\mathrm{Me}$ thode. Das Ergebnis hängt maßgeblich von einer explorationsfreudigen methodischen Gestaltung ab. Der berufliche Werdegang oder die Betrachtung bestimmter Wissensgebiete können als Zugang genutzt werden. Hilfreich ist eine Strukturierung der Wissensgebiete nach dem Grad der Explizierbarkeit: Fach- und Prozesswissen, Informationszugänge und Beziehungen, Kompetenzen. Aus den ausgewählten Themen werden die entsprechenden Lerninhalte benannt: Fallbeispiele, spezifische Arbeitsprozesse und Arbeitsroutinen, ausgewählte Dokumente und Statusberichte. Visualisierungen mit Hilfe von Skalen, Mindmaps, Skizzen und anderen Medien sind hierbei sehr hilfreich. Nachdem die Themen mit dem Leaving-Expert eruiert und vorpriorisiert wurden, werden sie mit den übrigen Prozessbeteiligten abgeglichen, also ihre Relevanz und der Bedarf für den Nachfolger werden ermittelt. Auf diese Weise werden sowohl Redundanzen als auch Lücken vermieden. Das vorhandene Wissen kann in einer Wissensstrukturkarte inventarisiert werden. Zur Vorbereitung des Wissenstransfers empfiehlt sich zudem ein »Transferplan «, in dem die Antworten zu den Fragen »Welches Wissen? Wem? Wie? Wann?« dem situativen Bedarf gemäß aufbereitet sind. Der Transfer- und der Wissensstrukturplan dienen der Dokumentation sowie dem Planen und Überprüfen des Übergabeprozesses.

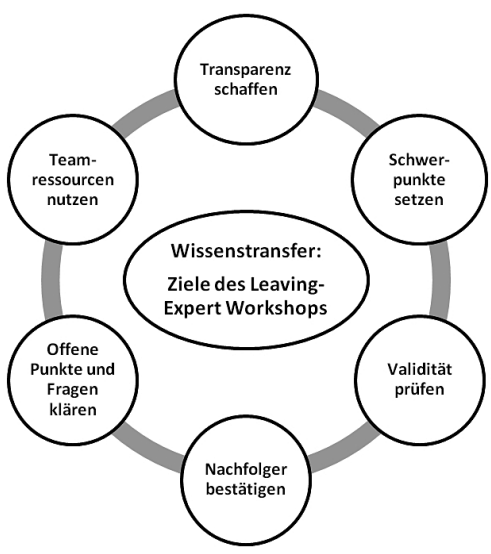

Abb. 2: Der Leaving-Expert-Workshop sollte sich auf sechs Hauptziele konzentrieren. 


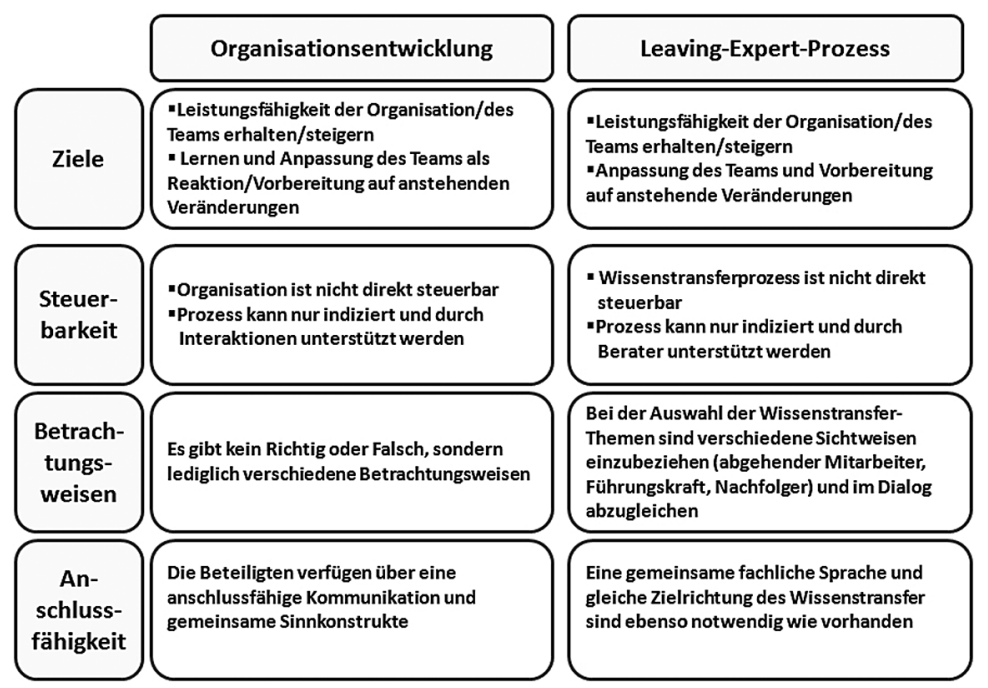

Abb. 3: Wenn Experten ausscheiden, erfordert dies stets eine Neuorientierung des Teams und der Einrichtung, selbst wenn die freiwerdende Stelle besetzt und als solche gleich bleibt.

- Teamphase: Zu Beginn der Teamphase wird die visualisierte Wissensstruktur kommuniziert. Indem weitere Prozessbeteiligte mit den bisherigen Ergebnissen bekanntgemacht werden, wird die Qualität der weiteren Planung sichergestellt. Dies erfolgt in idealer Weise in einem Workshop, in dem die verschiedenen Aspekte für den Wissenstransfer (vgl. Abb. 2) gemeinsam betrachtet werden. Hier entscheidet sich, ob es im Team zu einem Kompetenzzuwachs kommt. Deshalb wird dieser Phase eine hohe Aufmerksamkeit geschenkt. In den Workshop lässt sich - sofern gewünscht - der Aspekt der Teamentwicklung integrieren. Das Team wird auf die Zeit nach dem Weggang des scheidenden Mitarbeiters vorbereitet. Strukturen und Prozesse werden der neuen Situation angepasst, Rollen neu zugeschnitten, Vergangenes wird gewürdigt und Raum für Neues eröffnet.

- Transferphase: Die Transferphase beinhaltet den Wissenstransfer und den Kompetenzaufbau bei den Nachfolger. Hier spielt die Auswahl des Vorgehens eine maßgebliche Rolle dafür, ob der Transfer gelingt und es tatsächlich zum Kompetenzerwerb kommt. Es gibt eine Vielzahl bewährter Methoden, um Wissen zu transferieren. Diese reichen von selbstgesteuertem Lernen über rezeptive Unterrichtsformen bis $\mathrm{zu}$ intensiven Lerngruppen oder Meister-Lehrlings-Beziehungen.
Die Kompetenzmessung bildet den Abschluss dieser Phase oder indiziert, wenn eine weitere Kompetenzaufbauschleife eingeschoben werden muss. Die Effizienz des Kompetenzaufbaus kann durch didaktische Begleitung gefördert werden. Möglichkeiten hierfür sind Trainings zum Aufbau didaktischer Kompetenz oder die Moderation von Lerngruppen durch den Berater

\section{Was der Leaving-Expert- Prozess nützt}

Die Einführung einer Systematik für einen Leaving-Expert-Prozess enthält Chancen und Potenziale für das Unternehmen, die aus der Prozessbeschreibung zu erkennen sind. Er liefert eine solide Basis dafür, dass es zu einer tatsächlich nützlichen - weil in das Handeln integrierten - Weitergabe betriebsrelevanter Erfahrungen und Kompetenzen kommen kann. Nicht zuletzt hier zeigt sich, inwieweit Betriebe auch Nachfolge- und Einarbeitungsprozesse ernstnehmen.

So gesehen verhindert ein aktiv begleiteter Leaving-Expert-Prozess, dass der über eine lange Berufskarriere aufgebaute Erfahrungsschatz eines abgehenden Mitarbeiters verloren geht. Die enge Verzahnung des Leaving-Expert-Prozesses mit einem Prozess zur Team- und Organisationsentwicklung wurde bereits angesprochen. Scheiden Experten aus, bedeutet dies stets auch eine Veränderung des Teams oder der Betriebseinheit, selbst wenn die Stelle als solche gleich bleibt. Dieser Übergang bedeutet, dass Kompetenzen im Team neu hinzukommen oder aufgebaut werden müssen, und ist daher unserer Ansicht nach vor allem auch als Lernprozess zu begreifen und zu gestalten (vgl. Abb. 3).

$\mathrm{Ob}$ diese Lerngelegenheit als solche gesehen wird, zeigt sich u. a. darin, inwieweit ein Konzept für einen LeavingExpert-Prozess implementiert ist und systematisch, in Ansätzen oder Auszügen, je nach betrieblicher Erfordernis gelebt wird. In jedem Fall ist der Umgang mit ausscheidenden Expertinnen und Experten ein Indiz für die Wertschätzung des individuell Geleisteten. Daran werden sich Unternehmen zukünftig selbst erinnern, wenn sie ausgeschiedene Mitarbeitende als Berater wiedergewinnen wollen. Ein weiterer erwähnenswerter Aspekt ist die Integration des LeavingExpert-Prozesses in ein übergeordnetes Wissensmanagementkonzept.

Das Ausscheiden bedeutet auch, voneinander loslassen zu können und frei zu werden für einen neuen Abschnitt. Ein gelungener Übergang bedeutet einen Benefit für beide Seiten.

\section{Literatur}

Fischer, P. (2007). Berufserfahrung älterer Führungskräfte als Ressource. Wiesbaden. Königswieser, R./Hillebrand, M. (2009). Einführung in die systemische Organisationsberatung. 5. Aufl. Heidelberg.

Morschhäuser, M. (2006). Reife Leistung.

Personal- und Qualifizierungspolitik für die zukünftige Altersstruktur. Berlin.

Neuweg, G. (2004). Könnerschaft und implizites Wissen. Zur lerntheoretischen Bedeutung der Erkenntnis- und Wissenstheorie Michael

Polanyis. 3. Aufl. Münster.

Schultz-Wild, L./Böhle, F. (2006). Mit Verstand und allen Sinnen. Arbeiten im turbulenten Umfeld. Was erfolgreiche Profis "anders" machen. Bielefeld

Seyfried, B. (2011). Ältere Beschäftigte: Zu jung, um alt zu sein. Konzepte. Forschungsergebnisse. Instrumente. Bielefeld.

Statistisches Bundesamt (Hg.) (2009). 12. koordinierte Bevölkerungsvorausberechnung. [https://www.destatis.de/bevoelkerungspyramide, abgerufen am 6.3.2013]

Taudt, Ch. (2013). Wissenstransfer bei Verrentung von Mitarbeitern sicherstellen. Konzept eines Leaving-Expert-Prozesses. Berlin. 